UCRL $-C R--104838$

DE91 000952

NONDESTRUCTIVE EVALUATION OF RESIDUAL STRESS

IN ANISOTROPIC MATERIALS

Final Report

for

LLNL Intramural Order No. 2698203

George C. Johnson

Principal Investigator

Department of Mechanical Engineering

University of California

Berkeley, CA 94720

May, 1990

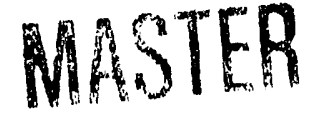

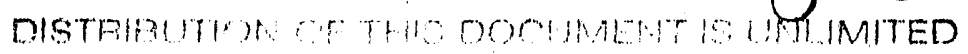




\section{DISC IAINIIR}

Work performed under the auspices of the (Is. Department of linergy by lawrence livermore National Iabora tory under contract mumber W-7405-6iNG;-48.

This document was prepared as an account of work sponsored by an agency of the l'nited States Government. Veither the l'nited Sitates (iovermment nor the l'niversity of California nor any of their empleyeeses, makes any warranty copress or implied, or assumes any legall lability or responsibility for the accuracy, completene'ss, or usefulness of any information, apparatus, product, or process disclosed, or represents that its use would not infringe prisately awned rights. Roference herein to iny specific commercial products, process, or service by trade name, trademark, manufacturer, or otherwise, does not necessurily constitute or imply its endorsement, recommendation, or favoring by the l'nited States Govermment or the Iniversity of california. The liews and opinions of anthors expressed herein do not neesessarily state or reflect those of the Linted States fowernment or the Iniversity of California, and shall not be used for advertising or prodent endorsement purposes. 


\section{INTRODUCTION}

The research supported under Lawrence Livermore National Laboratory Intramural Order Number 2698203, Project Title "Nondestructive Evaluation of Residual Stresses in Anisotropic Materials," focused on the ability to use a new nondestructive method for evaluating residual stresses in a wide range of materials which might exhibit anisotropy in their response. This new technique falls within the family of ultrasonic techniques referred to as acoustoelasticity.

Acoustoelasticity is an ultrasonic technique for stress evaluation which is based on the fact that the speeds at which various waves travel through a deformed body depend upon the state of stress to which the body is subjected. The basic approach has been to try to obtain estimates of the stress state from sufficiently precise measurements of the velocity variations.

The work supported by this contract has conclusively demonstrated that measurements of the variations in the speed at which longitudinal waves travel through a stressed body are sufficient to evaluate the complete state of stress within the body, provided certain conditions are met.

The specific focus on anisctropic materials in the research was addressed in a manner which was primarily analytical. All of the experiments involving materials with substantial elastic or plastic anisotropy indicated that the materials in question exhibited a level of acoustoelastic response which was at or below the limit for useful stress evaluation. Nevertheless, the analysis performed indicates that if an experimental system is built which allows determination of the velocity variation roughly an order of magnitude more precisely than is possible with the system used in this work, the complete state of residual stress may be obtained, despite the presence of anisotropy.

The remainder of this report consists of a detailed description of the technique and experimental system proposed for the evaluation of residual stress states. The underlying analytical developments are reviewed, and a numerical investigation into the application of this approach for anisotropic materials is presented. It is shown that an accurate assessment of the complete residual stress state may be obtained even in cases of extreme anisotropy. Finally, an experimental investigation of the technique is presented in which the experimentally determined stress state is compared with that predicted numerically. It is shown that the two estimates of stress agree well for the material involved. 


\section{OVERVIEW OF NONDESTRUCTIVE METHODS FOR STRESS EVALUATION}

Experimental methods for the determination of residual stresses in structural components have been the focus of considerable attention through the past several decades. A widely used class of techniques involves either destructive (parting and sectioning techniques) or semidestructive (blinc-hole drilling) methods. In addition to leaving the part examined unfit for service, these techniques require substantial expertise and are fairly costly to perform. A range of nondestructive techniques for stress evaluation have also been developed. One major technique involves diffraction of $x$-ray or neutron beams as a method of determining the strain on a particular lattice plane of the material. The physics of these processes is well understood and both diffraction techniques are capable of good spatial resolution, although the $x$-ray technique is limited to measuring the stresses near the surface. Neutrons are more deeply penetrating, but require the presence of a high flux reactor. Thus, there are relatively few facilities that can perform stress evaluation from neutron diffraction measurements.

An alternate nondestructive technique, acoustoelasticity, involves the measurement of the variation of speeds of ultrasonic waves caused by the presenc, of the stress field. Within the broad heading of acoustoelasticity, there are a range of diffe ent methods that have been considered, all of which are limited to the evaluation of plane states of stress. The most common acoustoelastic technique is is called the birefringence technique(Hsu, 1974; Fukuoka, et al., 1983; Pao, et al., 1984). This technique is based on the fact that for an initially isotropic material, the difference in the speeds at which two shear waves propagating normal to the plane of stress, but polarized in the principal stress directions, is proportional to the difference in the principal stresses. The constant of proportionality is a material constant (called the acoustoelastic constant for birefringence). Another technique which is currently receiving considerable attention involves the difference in the speeds of two SH waves propagating in one principal direction and polarized in the other (King and Fortunko, 1983; Thompson, et al., 1986; Man and $\mathrm{Lu}, 1987)$. In this case, the difference in the square of the SH wave speeds is equal to the difference in principal stresses divided by the material's mass density. There is no acoustoelastic constant which must be determined a priori for the $\mathrm{SH}$ wave technique. A third technique, called the longitudinal wave technique, involves the change in the speed of a longitudinal wave traveling in the direction normal to the plane of the stress (Kino, et al., 1979). This technique, like the birefringence technique, requires that the acoustoelastic constant be known in advance.

Each of the three acoustoelastic techniques discussed in the previous paragraph has certain advantages and disadvantages. A clear advantage of the SH wave technique is the absence of an acoustoelastic constant whose uncertainty affects the precision of the resulting stresses. The 
birefringence technique has an advantage in that there is a relatively larger velocity variation per unit stress than in either of the other techniques. The advantage of the longitudinal wave technique is the ease with which measurements can be made over a large region of a sample, and the spatial resolution which can be achieved. As will be shown later in this report, all of the techniques use relative measurements (as opposed to absolute measurements) of velocity variation.

The remainder of this report addresses the application of the longitudinal wave technique to evaluating the residual stress state throughout a sample. A recent analytical development has made it possible to estimate the complete residual stress state (both normal and shear stress components) everywhere in a planar structure (Johnson and Dike, 1988). Neither of the other acoustoelastic techniques have yet to be demonstrated as having the capability for such whole-field stress determination.

In the next section, the basic theory is presented for the stress evaluation from measurements of variations in longitudinal wave speeds. While the technique should provide exact results for the case of an isotropic material (given perfect data), we consider also the more realistic case of a material which exhibits acoustoelastic anisotropy. Experimental considerations are presented in Section 4, where we provide new equations for the exact determination of spatial velocity variations from measurements of variations in time-of-flight and sample thickness. Section 5 presents certain numerical considerations which must be taken into account in solving the system of equations given the limitations of the measu ed data. It is shown here that the technique for dealing with the anisotropy provides the corr ct stress solution, even for rather extreme cases of anisotropy. Finally, experimental results fo the residual stresses in an aluminum ring are presented and compared with numerical estimates of the stress state. It is shown that both the spatial variations and the magnitudes of the experimental and numerical estimates are in good agreement.

\section{THEORY FOR STRESS EVALUATION USING LONGITUDINAL WAVES}

Consider a body subject to a plane state of residual stress, with Cartesian components $\sigma_{x x}$, $\sigma_{y y}$, and $\sigma_{x y}$, through which a longitudinal wave propagates in the direction normal to the plane. The material in question is taken to be initially homogeneous and acoustoelastically orthotropic, so that the shift in the speed $V$ of this longitudinal wave from the speed $V_{o}$ in the unstressed material is (King and Fortunko, 1983; Johnson and Mase, 1984)

$$
\frac{V-V_{o}}{V_{o}}=A_{x} \sigma_{x x}+A_{y} \sigma_{y y}
$$


where $A_{x}$ and $A_{y}$ are the acoustoelastic constants, which may be different for an anisotropic material. An alternate form of this equation, which may be more revealing in terms of the eventual stress evaluation, is

$$
\frac{V-V_{o}}{V_{o}}=A_{+}\left(\sigma_{x x}+\sigma_{y y}\right)+A_{-}\left(\sigma_{x x}-\sigma_{y y}\right)
$$

where

$$
A_{+}=1 / 2\left(A_{x}+A_{y}\right)+A_{-}=1 / 2\left(A_{x}-A_{y}\right)
$$

Note that for a material which is acoustoelastically isotropic, $A_{-}=0$ and the change in wave speed from the unstressed state is proportional to the sum of the in-plane normal stresses. In most engineering materials, the magnitude of $A_{+}$is considerably greater than that of $A_{-}$, so that the material can often be considered as being "slightly anisotropic". Although we do not impose a condition of such slight anisotropy, we do assume that $\left|A_{+}\right|>\left|A_{-}\right|$and that the values of the acoustoelastic constants are known.

The equilibrium equations for the residual stress field in the absence of body forces are

$$
\begin{aligned}
& \sigma_{x x, x}+\sigma_{x y, y}=0, \\
& \sigma_{x y, x}+\sigma_{y y, y}=0,
\end{aligned}
$$

where comma denotes partial differentiation with respect to the indicated coordinate. An equation for the shear stress $\sigma_{x y}$ in terms of the normal stresses may be obtained by differentiating Eq. (4) 1 with respect to $y$ and $E q$. (4) 2 with respect to $x$, and adding. Thus,

$$
\nabla^{2} \sigma_{x y}=-\left(\sigma_{x x}+\sigma_{y y}\right), x y
$$

where $\nabla^{2}$ is the two-dimensional Laplace operator. Equation (5) is a Poisson's equation for the shear stress in terms of the mixed derivative of the surn of the normal stresses.

If an accurate estimate of the sum of the normal stresses can be obtained from acoustoelastic measurements, then Eqs. (4) and (5) can be solved for the entire stress field in the body. If $A_{-}$ $=0$ (acoustoelastic isotropy), the right-hand side of Eq. (5) is directly related to the acoustoelastic measurements. Let us now focus attention on the problem posed by a material which is acoustoelastically anisotropic.

In order to find a solution for the shear stress by integration of Eq. (5), the values of $\sigma_{x y}$ along the boundary must be known. Because the stresses are residual, the boundaries are taken to be traction free. Consider a point on the boundary with outward unit normal vector $\mathbf{n}$ which makes an angle $\theta$ with the $x$-axis. The stress tensor at this point may be expressed either in 
terms of the Cartesian $(x-y)$ components used above, or in terms of normal-tangential (n-t) components $\sigma_{n n}, \sigma_{t}$, and $\sigma_{n t}$, which are related to the Cartesian components as

$$
\begin{aligned}
& \sigma_{n n}=\sigma_{x x} \cos ^{2} \theta+\sigma_{y y} \sin ^{2} \theta+\sigma_{x y} \sin 2 \theta \\
& \sigma_{t t}=\sigma_{x x} \sin ^{2} \theta+\sigma_{y y} \cos ^{2} \theta-\sigma_{x y} \sin 2 \theta \\
& \sigma_{n t}=1 / 2\left(\sigma_{y y}-\sigma_{x x}\right) \sin 2 \theta+\sigma_{x y} \cos 2 \theta
\end{aligned}
$$

In the case considered here (traction-free boundaries), the only nonvanishing stress component in normal-tangential coordinates is $\sigma_{t t}$. Thus, the Cartesian components are expressed in terms of $\sigma_{t}$ as

$$
\sigma_{x x}=\sigma_{t t} \sin ^{2} \theta, \quad \sigma_{y y}=\sigma_{t t} \cos ^{2} \theta, \quad \sigma_{x y}=-1 / 2 \sigma_{t l} \sin 2 \theta
$$

In light of Eq. (1), the velocity change from the unistressed state is related to the tangential component of stress as

$$
\frac{V-V_{o}}{V_{o}}=\sigma_{t t}\left(A_{x} \sin ^{2} \theta+A_{y} \cos ^{2} \theta\right)
$$

Assuming that measurements of velocity change can be made along the boundary and that the geometry of the boundary $(\theta)$ is known, the shear stress $\sigma_{x y}$ can be determined through Eqs. $(7)_{3}$ and (8).

Unfortunately, because the material is not isotropic, we cannot obtain the right-hand side of Eq. (5) directly from the measurements. Instead, we propose to use an iterative scheme in which the velocity data is used to provide an estimate of $\sigma_{x x}+\sigma_{y y}$ which is updated at the end of each step of the iteration. Specifically, the initial estimate of the sum of the stresses is ohtained by letting $A_{\text {- }}$ be zero in Eq. (2). The boundary values for $\sigma_{x y}$ and this initial guess are used to solve for the shear stress throughout the sample. The equilibrium equations are then used to estimate the normal stresses. At this point, we have estimates of $\sigma_{x x}, \sigma_{y y}$, and $\sigma_{x y}$ which are not consistent with Eq. (2). However, by using these estimates and the actual value of $A_{-}$, we obtain at the end of each step of the iteration, a new estimate of the sum of the stresses through the equation

$$
\left(\sigma_{x x}+\sigma_{y y}\right)_{n+1}=\frac{1}{A_{+}}\left[\frac{V-V_{o}}{V_{o}}-A_{-}\left(\sigma_{x x}-\sigma_{y y}\right)_{n}\right]
$$

where the subscripts " $n$ " and " $n+1$ " refer to the iteration steps involved. It is shown later that this scheme converges for synthetic data to the actual stress field, even for fairly extreme levels of anisotropy. 


\section{EXPERIMENTAL PROCEDURES}

The algorithm presented in the preceding section assumes that sufficiently precise estimates of the velocity shift with stress can be experimentally determined. Velocity is not, however, a directly measurable quantity. Further, we would like to be able to evaluate the residual stress state without prior knowledge of the unstressed velocity $V_{o}$. We show in this section that the use of two scans which provide spatial variations in the time-of-flight and path length of the longitudinal waves are sufficient to obtain the necessary velocity shift.

It is impurtant to recognize that there are two types of variations or shifts involved in the experimental work. We refer to these as configurational variations and spatial variations. For the velocities, these variations are associated with the following definitions:

$\Delta V / V_{o}$ is the configurational velocity change. It is the relative velocity change between the final (stressed) and initial (unstressed) configurations, at the same point.

$\delta V / V$ is the spatial velocity variation. It is the relative velocity difference between two points in the final configuration.

The configurational velocity change is the quantity which is needed for the stress evaluation, while spatial variations of the thickness and time-of-flight are actually measured.

In keeping with our previous usage, we let a subscript o denote quantities associated with the initial (unstressed) configuration. Symbols written without a subscript are taken to represent quantities in the final (stressed) configuration. In order to discuss the spatial variation of a quantity, we let $X$ denote any generic material point, and $X$, denote a specific reference point. Thus, the velocities in the initial configuration would be written $V_{o}(X)=V_{o}\left(X_{r}\right)=V_{o}$, while the velocities in the final configuration would be written $V(X)$ and $V\left(X_{r}\right)$, where these two velocities would in general be different. For simplicity, we often omit the argument $X$ for the generic material point (so that $V(X)$ would be written simply as $V$ ).

The configurational velocity change is then just

$$
\frac{\Delta V}{V_{o}}=\frac{V-V_{o}}{V_{o}}
$$

while the spatial variation is expressed as

$$
\frac{\delta V}{V}=\frac{V-V\left(X_{r}\right)}{V\left(X_{r}\right)} .
$$


The measurements made are of two types: spatial variations of the time "of-flight of the longitudinal wave, and spatial variations of the thickness of the sample (which is the path length of the wave). The system used for the time-of-flight variations is a slight modification of the double-pulse overlap system described by Ilic', et al. (1979). In this system, a single transducer operates in a water-bath and is excited by two of tone bursts which are timed so that the first echo caused by the second pulse and the second echo caused by the first pulse return to the transducer at the same time. Both torie bursts have the same carrier frequency (typically around $10 \mathrm{MHz}$ ) which can be adjusted to provide a particular phase delay between the two overlapping echoes. By using the analog phase-lock loop described by Ilic', et al. (1979), this phase delay can be held constant as the time-of-flight changes due to changes in the wave speed and sample thickmess. The condition at which such a phase condition is achieved is called a "null" and the associated frequency is denoted $f_{T}$, with the subscript indicating that this frequency is related to the timeof-flight.

The system used for the measurement of thickness variation is the two-transducer system proposed by Fisher and Johnson (1984). The two transducers are collinear and are mounted pointing directly at one another on a "rigid" fixture. The sample is placed between the transducers with its major surfaces normal to the axis of the transducers. The fixed distance between the transducers is $L$, while the distance from each transducer to the nearest face of the sample is $l_{1}$ or $l_{2}$. Each transducer is excited by a single if tone burst. If we consider only the first echo returning to each transducer, the total phase delay for each wave is

$$
\phi_{\alpha}=\frac{f_{d} l_{\alpha}}{V_{w}}, \alpha=1,2
$$

where $f_{d}$ is the carrier frequency of the tone bursts and $V_{w}$ is the speed at which the waves travel in the water. Adding the two phases, and noting that $L=l_{1}+l_{2}+d$, gives

$$
\Phi=\phi_{1}+\phi_{2}=\frac{2 f_{d}}{V_{w}}\left(l_{1}+l_{2}\right)=\frac{2 f_{d}}{V_{w}}(L-d) .
$$

This phase sum can be held constant for changing thickness by varying the frequency $f_{d}$. Thus, if $V_{w}$ is taken to be a constant, the variation in thickness can be related to the variation in frequency through the relation

$$
\frac{\delta d}{d}\left[1+\frac{\delta f_{d}}{f_{d}}\right]-\frac{L-d_{r}}{d_{r}} \frac{\delta f_{d}}{f_{d}}=0,
$$

where the symbol $\delta$ is used to denote the spatial nature of the variations, and $d_{r}$ is the thickness of the sample at $X_{r}$. We note that this expressicn is exact, while the associated relation given by Fisher and Johnson (1984), ignores the term which is nonlinear in the variations. 
Given this method for determining the spatial thickness variation, let us return to the single transducer systern. The phase delay $\phi$ between the two echoes may be written as the product of the frequency and the time-of-flight. Alternatively, the time-of-flight may be eliminated in favor of the thickness and velocity, so that the phase delay becomes

$$
\phi=\frac{2 f_{T} d}{V} .
$$

Since $\phi$ is held constant at a particular null, the spatial velocity variation is related to the spatial variations in null frequency and thickness as

$$
\frac{\delta V}{V}=\frac{\delta d}{d}+\frac{\delta f_{T}}{f_{T}}+\frac{\delta d}{d} \frac{\delta f_{T}}{f_{T}},
$$

where this expression is again exact.

The importance of retaining the exact expressions in Eqs. (14) and (16) is clear when the acoustoelastic constants of the material being investigated are stnall. In such cases, the maximum velocity change may be on the order of $0.1 \%$, while the variations in thickness and null frequency may be much larger (on the order of several percent) and of the opposite sign. The terms which are nonlinear in the variations in these equations may then contribute substantially to the resulting velocity variation.

We now have a method with which to determine the spatial variation in the velocity, but in fact need the configurational variation in order to evaluate the stresses. To obtain this latter variation, we make use of the fact that the residual stress field must be self-equilibrating. Specifically, the volume integrals of the normal stress components over the entire region must vanish. Since we are dealing with the case of plane stress, these integrals over the volume can be replaced by integrals over the surface of the sample. Given Eqs. (1) or (2), the vanishing of these surface integrals then requires that the integral of the configurational velocity change over the sample must be zero.

Let us now consider the relation between the configurational and spatial variations of velocity. Equation (11) may be expanded as

$$
\frac{\delta V}{V}=\frac{V(X)-V_{o}}{V\left(X_{r}\right)}-\frac{V\left(X_{r}\right)-V_{o}}{V\left(X_{r}\right)}=\frac{V_{o}}{V\left(X_{r}\right)}\left[\frac{\Delta V}{V_{o}^{\prime}}-C\right],
$$

where $C$ is the configurational velocity change at the reference point and will be treated as a constant. The ratio of initial velocity to final velocity at the reference point is taken to be nearly unity (it rarely deviates from unity by more than $1 \%$ ) so that $\mathrm{Eq}$. (17) may be rewritten as

$$
\frac{\Delta V}{V_{o}}=\frac{\delta V}{V}+C \text {. }
$$


Since the integral of the left-hand side of Eq. (18) must be zero, the constant $C$ may be determined from the integral of the spatial variation over the surface area $\Sigma$ as

$$
C=-\frac{1}{\Sigma} \int \frac{\delta V}{V} d \Sigma
$$

Thus, the measurement of the spatial variations is sufficient to provide the necessary configurational data.

The electronic system used for the single transducer measurements is essentially that described by Ilic', et al. (1979). The electronic system for the two-transducer system is similar, although the analog signal processing is somewhat diffe rent. A schematic diagram of the system used for the thickness scans is shown in Fig. 1. In order to isolate $\Phi$, the sum of the individual phases, the rf tone burst which drives one of the transducers, say the second one, is modulated by a low frequency signal. Thus, the returning pulses $r_{\alpha}, \alpha=1,2$, are of the form

$$
r_{1}=B_{1} \cos \left(\omega_{d} t+\phi_{1}\right), \quad r_{2}=B_{2} \cos \left(\omega_{d} t+\phi_{2}\right) \cos \Omega t
$$

where $B_{\alpha}$ are the waves' amplitudes, $\omega_{d}=2 \pi f_{d}$, and $\Omega$ is the frequency of the modulation, which is typically in the low kilohertz range. These two signals are electronically mixed and the result is filtered to isolate the second harmonic of the carrier. This component of the signal has the form

$$
r_{3}=B_{3} \cos \left(2 \omega_{d} t+\Phi\right) \cos \Omega t
$$

This signal is then mixed with a continuous wave whose frequency is $2 \omega_{d}$. The amplitude of the low-frequency component of this final signal is proportional to $\cos \Phi$,

$$
r_{4}=B_{4} \cos \Phi \cos \Omega t
$$

Thus, when this signal is used as input to a lock-in amplifier whose reference frequency is $\Omega$, the output of the lock-in is a dc signal proportional to $\cos \Phi$. This dc signal is then integrated and the result is used to drive an FM modulator until the lock-in output reaches zero (a null condition).

The present system uses commercially available $10 \mathrm{MHz}$, spherically focused transducers with $50 \mathrm{~mm}$ ( $2 \mathrm{in}$.) focal lengths. In actually performing the scans for the thickness variation, we must choose the geometry of the transducer holder (spacing $L$ ) in recognition that there are two effects which compete with one another and which must be balanced. On the one hand, the use of distinct rf tone bursts for the phase comparison requires that the lengths $l_{\alpha}$ be sufficiently large, while on the other hand, large path lengths (large $L$ ) result in small frequency variations for a given thickness variation. 
Most of the measurements made to date have been on the samples that are approximately $10 \mathrm{~mm}$ thick with the sample surfaces between 25 and $40 \mathrm{~mm}$ from the transducers' faces. The result is somewhat poorer spatial resolution than would be expected if the transducers were operated at their focal lengths. If the transducers are used at their focal lengths, adjacent null frequencies are only $0.07 \%$ apart and the sensitivity of the thickness evaluation is poor. Thus, we can make measurements to within approximately $2 \mathrm{~mm}$ of the edge of the sample. Our current positioning system consists of two perpendicular lead screw stages driven by stepping motors, and has a nominal spatial resolution of $50 \mu \mathrm{m}$. When scans of the same region of a sample are repeated, it is found that the null frequencies for these systems have a repeatability of .005\% over a $1 \%$ maximum variation, as determined by the RMS difference in the null frequencies over all points of the scans.

\section{NUMERICAL PROCEDURES}

The basic equations have been cast into a finite difference scheme for the solution of the residual stress field. For the present work, we have'restricted attention to disks or annuli which are most conveniently described in plane polar coordinates. In this section we describe results obtained using synthetic velocity data generated from a known stress state. This numerical example does not use a residual stress state, but the requirement that the stresses be residual is not operative as long as the appropriate boundary values of $\sigma_{x y}$ and the initial velocity $V_{o}$ are known. We demonstrate that the proposed procedure for stress evaluation in materials which exhibit anisotropic acoustoelastic response converges, and that the solution is basically in agreement with the actual stress state. Experimental results for a residually stressed aluminum ring are presented in the following section.

We consider for this example the stress state generated by the far-field tension of an infinite plate of elasically isotropic material containing a circular hole. We know the exact stress state in terms of components expressed in either polar or Cartesian coordinates. Our approach is to use the known normal stresses in Eq. (1) to generate the synthetic velocity variations given various choices of acoustoelastic constants. The known shear stresses $\sigma_{x y}$ along the edges of an annular region are used with the velocity variations to estimate the stress state in the interior of the annulus.

Contours of the shear stress and the normal stress in the loading direction are shown in Fig. 2 for an annular region of the plate under a far-field tension of $100 \mathrm{MPa}$. The stresses displayed 
in Fig. 2 result from velocity data at discrete grid points within the interior of the region assuming that the material is acoustoelastically isotropic and that $A_{+}=10 \mathrm{TPa}^{-1}$ (typical of many aluminums). In the results shown, there are 9 radial locations between the inner and outer boundaries, and the grid points are spaced at $5^{\circ}$ intervals in the circumferential direction. This is a rather coarse grid (only 153 data points in the interior of the region), but it serves to show that the a'gorithm provides stress values which are everywhere within $5 \mathrm{MPa}$ of the exact values.

When the material is taken to be acoustoelastically anisotropic, the same stress state leads to a different velocity variation. However, the same stress pattern emerges after the iterative process described in Section 3. Table I gives the number of iterations required to reduce the maximum stress difference berween subsequent iterations to within $0.1 \mathrm{MPa}$ for a range of different anisotropies. We find that the technique converges for all cuses. In particular, we note that the last case considered is an extreme case of anisotropy in which $A_{x}=20 \mathrm{TPa}^{-1}$ and $A_{y}=0 \mathrm{TPa}^{-1}$. Despite this degree of anisotropy, the iterative procedure converged after only 14 iterations.

Table I. Number of iterations required for convergence in anisotropic materials. In all cases, $A_{+}=10 \mathrm{TPa}^{-1}$.

\begin{tabular}{cc}
\hline \hline$A_{-}\left(T P a^{-1}\right)$ & Iterations \\
\hline 1 & 3 \\
2 & 4 \\
4 & 5 \\
6 & 7 \\
8 & 9 \\
9 & 11 \\
10 & 14 \\
\hline \hline
\end{tabular}

\section{EXPERIMENTAL RESULTS}

An annulus of 6061-T6 aluminum, with nominal thickness of $12.7 \mathrm{~mm}$, inside diameter of $38.1 \mathrm{~mm}$, and outside diameter of $63.5 \mathrm{~mm}$, was loaded in diametral compression until permanently deformed anci then completely unloaded. Loading was performed using ball-in-socket compression platens acting on flat regions which had been machined on the top and bottom of the annulus. One quadrant of the specimen was scanned over a $1 \mathrm{~mm}$ radial, $2.5^{\circ}$ 
circumferential grid. Experimentally determined stress contours are compared with those estimated by the NIKE2D finite element code (Hallquist, 1986).

Because measurements cannoi be made at the very edge of the sample, the interior measurements are extrapolated to obtain the boundary values of the stresses. The extrapolation procedure used for the results shown below involved the least-squares fit of a straight line to the points near the boundary. The interior data is used where it is available, with extrapolated data used only where necessary.

Contours of residual pressure shown in Fig. 3 indicate generally good agreement between the experimental and numerical estimates. Note in particular the results of the two approaches for the zero contour $(\mathrm{C})$. While the experimental contour is somewhat noisier than the numerical contour, the overall agreement indicates that the method for evaluating the constant $C$ from Eq. (19), and so the sum of stresses, is valid. The fact that the experimental contours are noisier than the numerical contours is to be expected due to the intrinsic uncertainty in the measurements. This noise is especially noticeable in the low-stress regions of the annulus. We also note that the contours generally have the correct shape and are properly located spatially.

Figure 4 presents the experimental and numerical estimates of the shear stress $\sigma_{x y}$. Again, the zero-stress contour $(E)$ has the same basic pattern throughout the region and is noisier in the experimental plot. The regions of positive and negative shear are in uniform agreement, though there are again certain regions within which the magnitudes are somewhat different. Under the flat at the top, for example, the experimental contours accurately denote the stress concentration at the edge of the flat, but overpredict the magnitude of the shear stress at this point.

Figure 5 presents contours for the normal stress $\sigma_{x x}$, which is the hoop stress at the top of the sample. The region in which this stress component is small is accurately delineated and, as in the previous plots, the zero-stress contours (E) agree reasonably well. The regions of tension and compression are in spatial agreement, though the magnitudes of the experimental estimates are higher at the boundaries than are the numerical estimates.

Extrapolation tends to be an inherently inaccurate process and, as noted above, can cause difficulties at the boundaries. It was found that while various extrapolation procedures yielded large differences in the values of stresses at the boundary, the interior values were affected very little. The larger the ratio of area where measured data is available to that where extrapolated values must be used, the better the results of this method can be expected to be. 


\section{REFERENCES}

M.J. Fisher and G.C Johnson, "Acoustic Velocity Variations due to Finite Grain Size in Polycrystalline Materials," in Review of Progress in Quantitative NDE, edited by D.O. Thompson and D.E. Chimenti (Plenum Press, New York), Vol. 3B, pp. 1119-1128.

H. Fukuoka, H. Toda, and N. Naka, 1983, "Nondestructive Residual Stress Measurement in a Wide-Flanged Rolled Beam by Acoustoelasticity," Experimental Mechanics, Vol. 23, 120128.

J.O. Hallquist, 1979, NIKE2D - A Vectorized Implicit, Finite Deformation, Finite Element Code for Analyzing the Static and Dynamic Response of 2-D Solids with Interactive Rezoning and Graphics, Lawrence Livermore National Laboratory UCID No. 19677, Livermore, CA.

N.N Hsu, 1974, "Acoustical Birefringence and the Use of Ultrasonic Waves for Experimental Stress Analysis," Experimental Mechanics, Vol. 14, 169-176.

D.B. Ilic', G.S. Kino, and A.R. Selfridge, 1979, "Computer Controlled System for Measuring Two-Dimensional Velocity Fields," Review of Scientific Instrumentation, Vol. 50, 15271533.

G.C. Johnson and J.J. Dike, 1988, "Complete Evaluation of Residual Stress States using Acoustoelasticity," in Review of Progress in Quantitative NDE, edited by D.O. Thompson and D.E. Chimenti (Plenum Press, New York), Vol. 7B, pp. 1391-1398.

G.C. Johnson and G.T. Mase, 1984, "Acoustoelasticity in Transversely Isotropic Materials,", Journal of the Acoustical Society of America, Vol. 75, 1741-1747.

R.B. King and C.M. Fortunko, 1983, "Determination of in-plane Stress States in Plates Using Horizontally Polarized Shear Waves," Journal of Applied Physics, Vol. 54, 3027-3035.

G.S. Kino, J.B. Hunter, G.C. Johnson, A.R. Selfridge, D.M. Barnett, G. Herrmann, and C.R. Steele, 1979, "Acoustoelastic Imaging of Stress Fields," Journal of Applied Physics, Vol. $50,2607-2613$.

C.-S. Man and W.Y'. Lu, 1987, Towards an Acoustoelastic Theory for Measurement of Residual Stress", Journal of Elasticity, Vol. 17, 159- . 
Y.-H. Pao, W. Sachse, and H. Fukuoka, 1984, "Acoustoelasticity and Ultrasonic Measurements of Residual Stress," in Physical Acoustics, edited by W.P. Mason and R.N. Thurston (Academic Press, New York), Vol. XVII, Chap. 2, pp. 61-143.

R.B. Thompson, S.S. Lee, and J.F. Smith, 1986, "Angular Dependence of Ultrasonic Wave Propagation in a Stressed Orthorhombic Continuum: Theory and Application to the Measurement of Stress and Texture," Journal of the Acoustical Society of America, Vol. 80, 921931. 


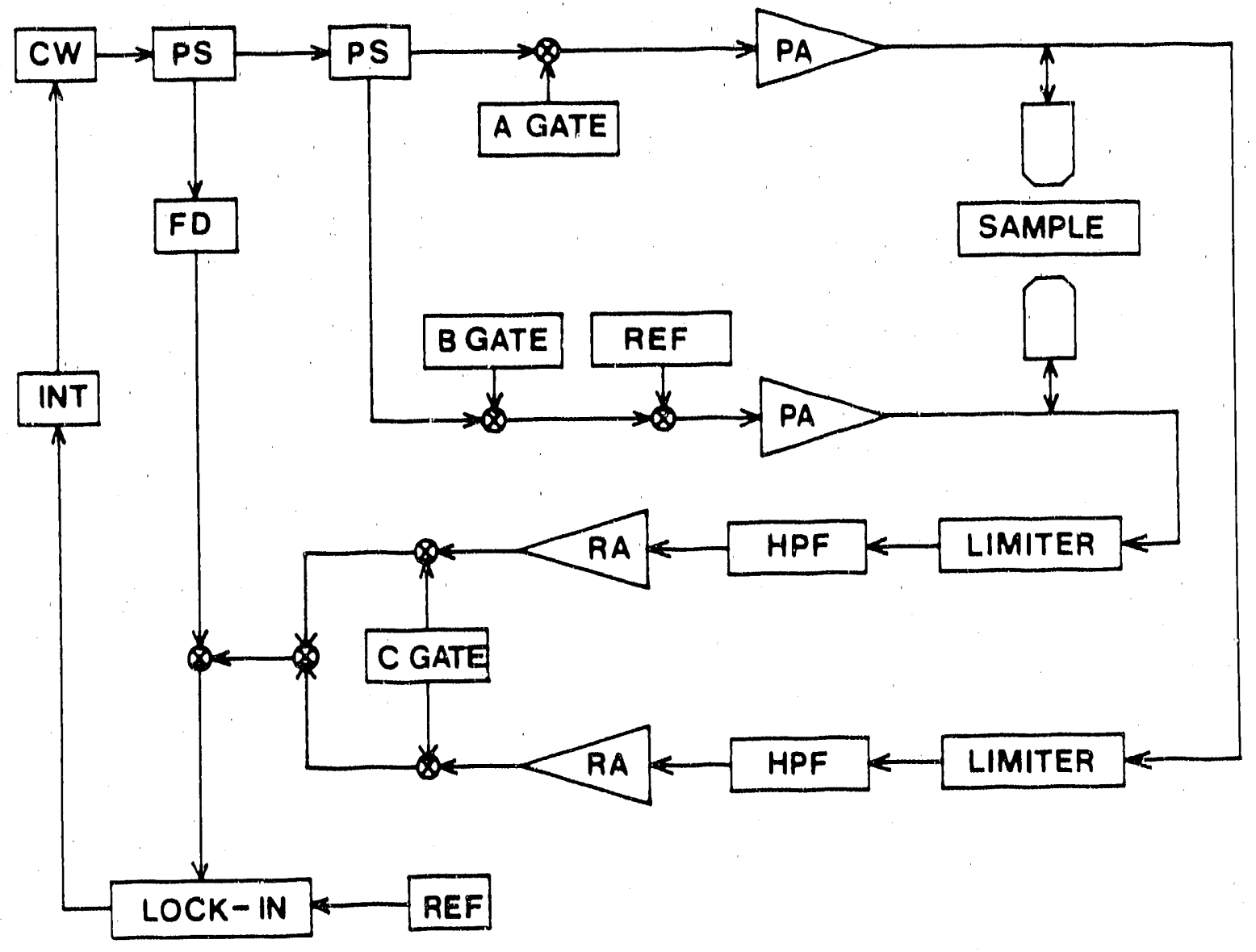

Figure 1. Electronic block diagram for the two-transducer thickness scanning system. 


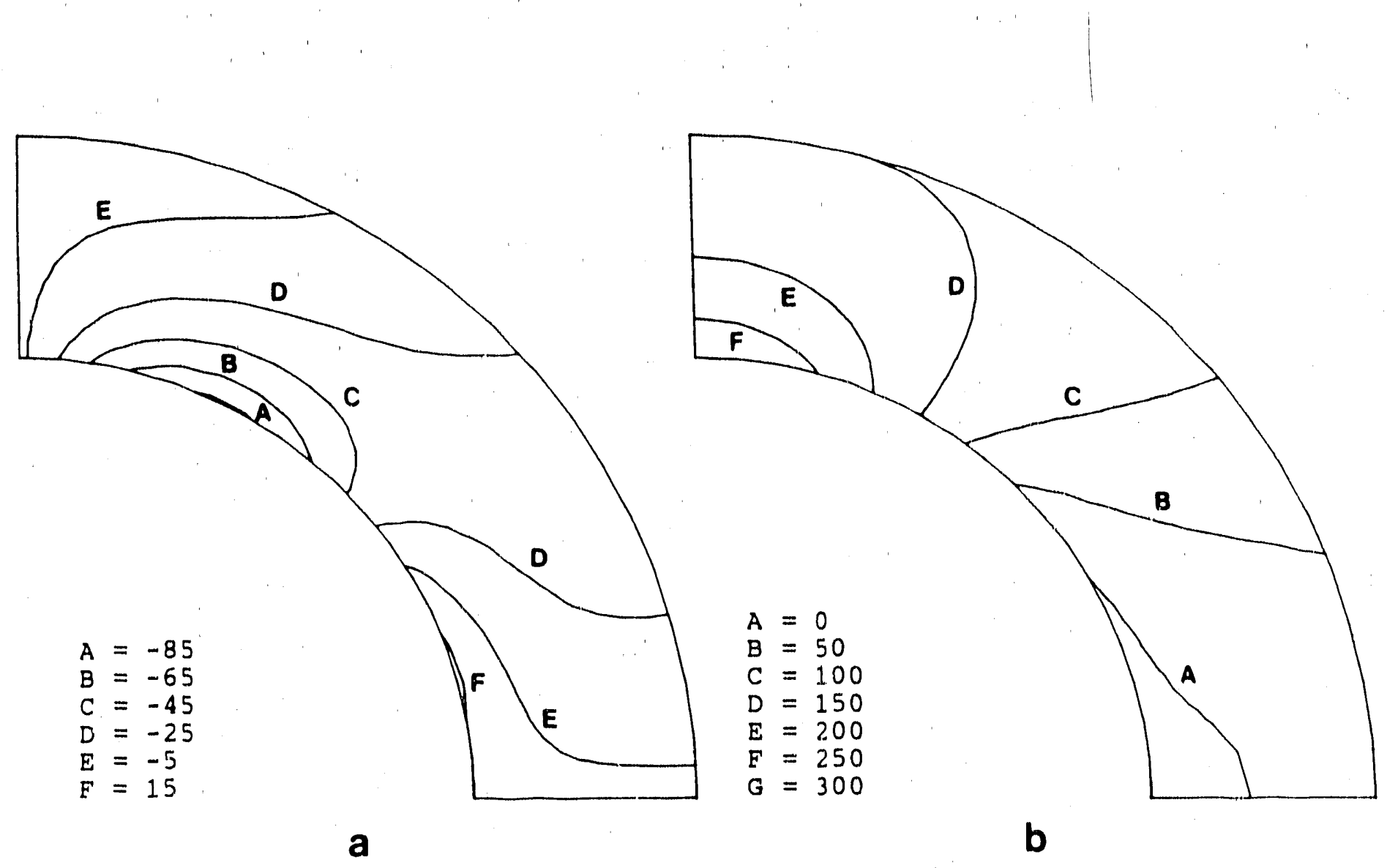

Figure 2. Contours of (a) shear stress $\sigma_{x y}$, and (b) normal stress $\sigma_{x x}$ in an annular region of an infinite plate subject to far-field tension in the $x$-direction of $100 \mathrm{MPa}$, obtained by numerically integrating Eqs. (4) and (5) given synthetic velocity data at the grid points. Contours are given in $\mathrm{MPa}$. 


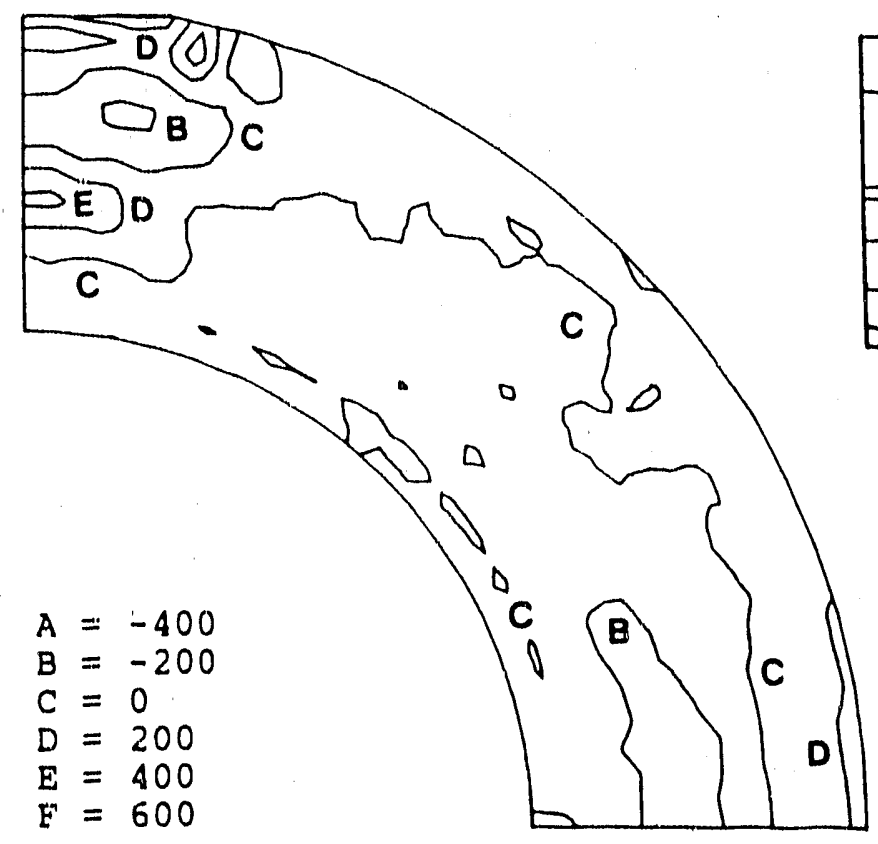

a

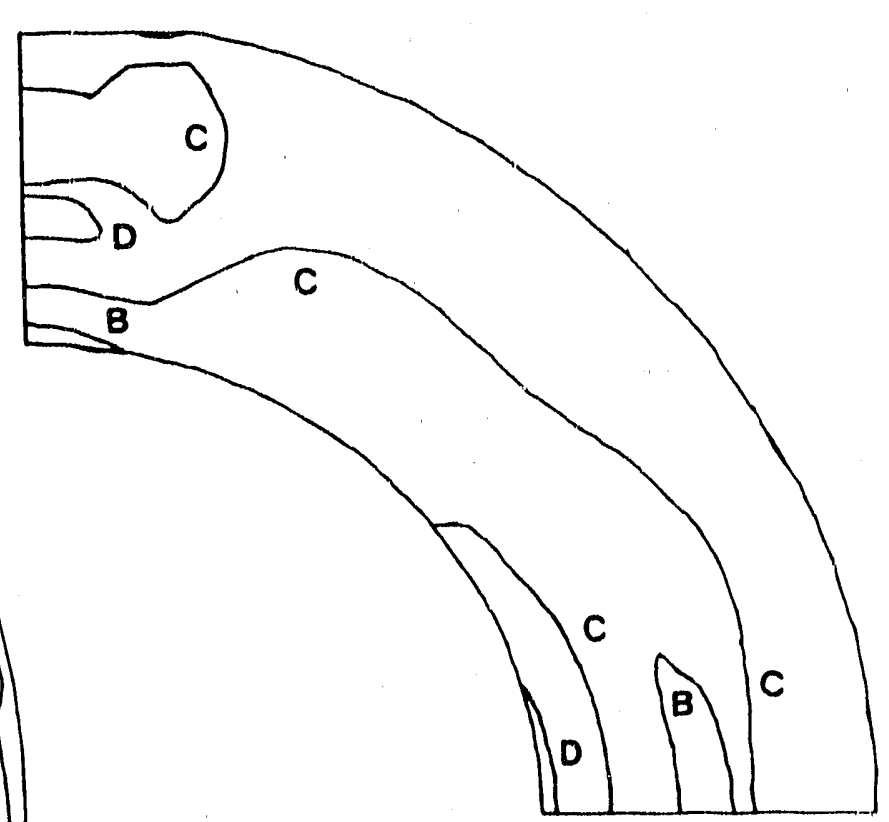

b

Figure 3. Contours of the sum of normal residual stresses $\sigma_{x x}+\sigma_{y y}$ obtained (a) experimentally and (b) numerically for a 6061-T6 aluminum ring subject to diametral compression and unloaded. Contours are given in MPa. 

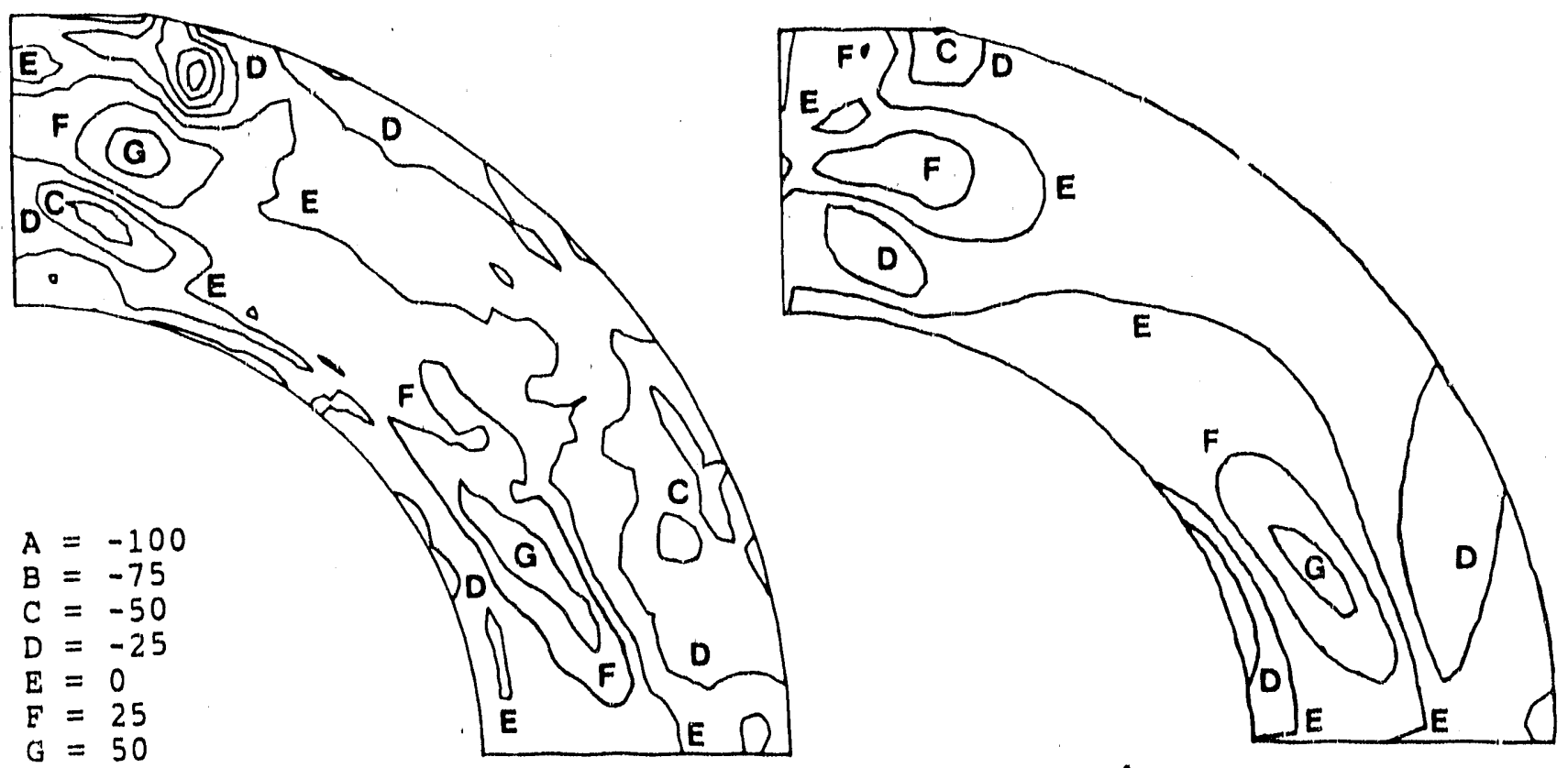

a

b

Figure 4. Contours of the residual shear stress $\sigma_{x y}$ obtained (a) experimentally and (b) numerically for a 6061-T6 aluminum ring subject to diametral compression and unloaded. Contours are given in $\mathrm{MPa}$. 


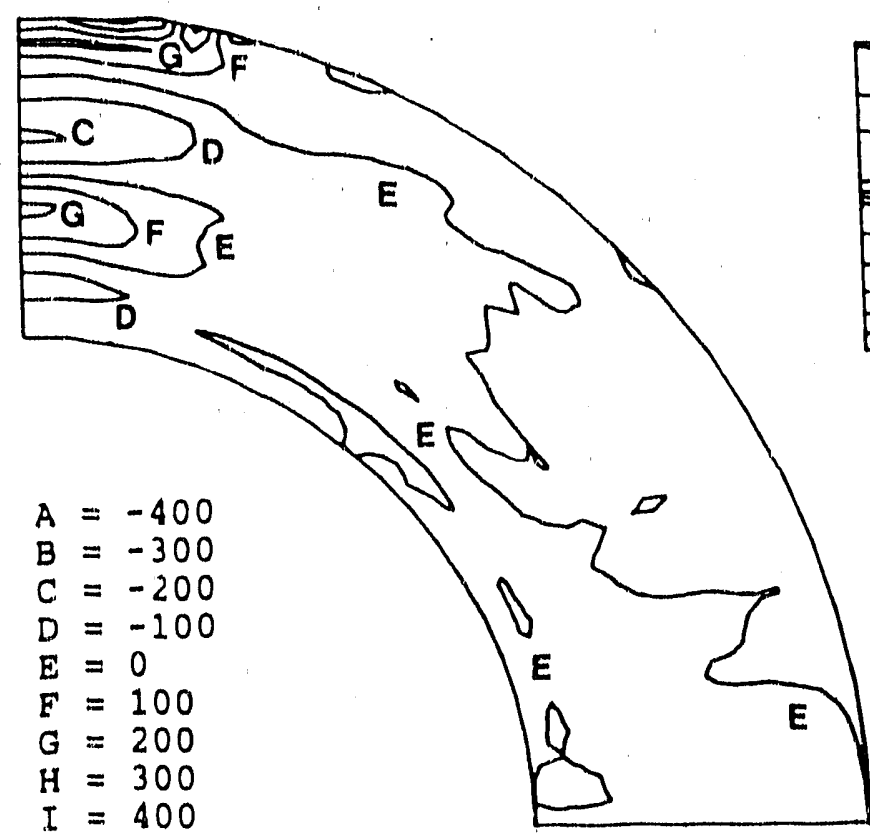

a

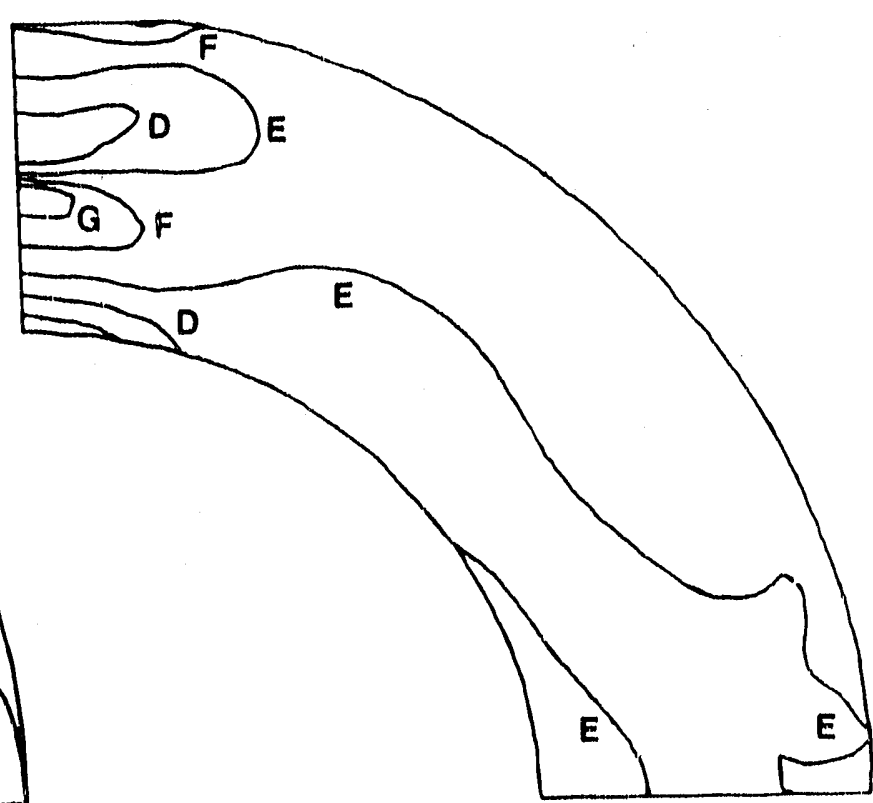

b

Figure 5. Contours of the residual normal stress $\sigma_{x x}$ obtained (a) experimentally and (b) numerically for a 6061-T6 aluminum ring subject to diametral compression and unloaded. Contours are given in $\mathrm{MPa}$. 

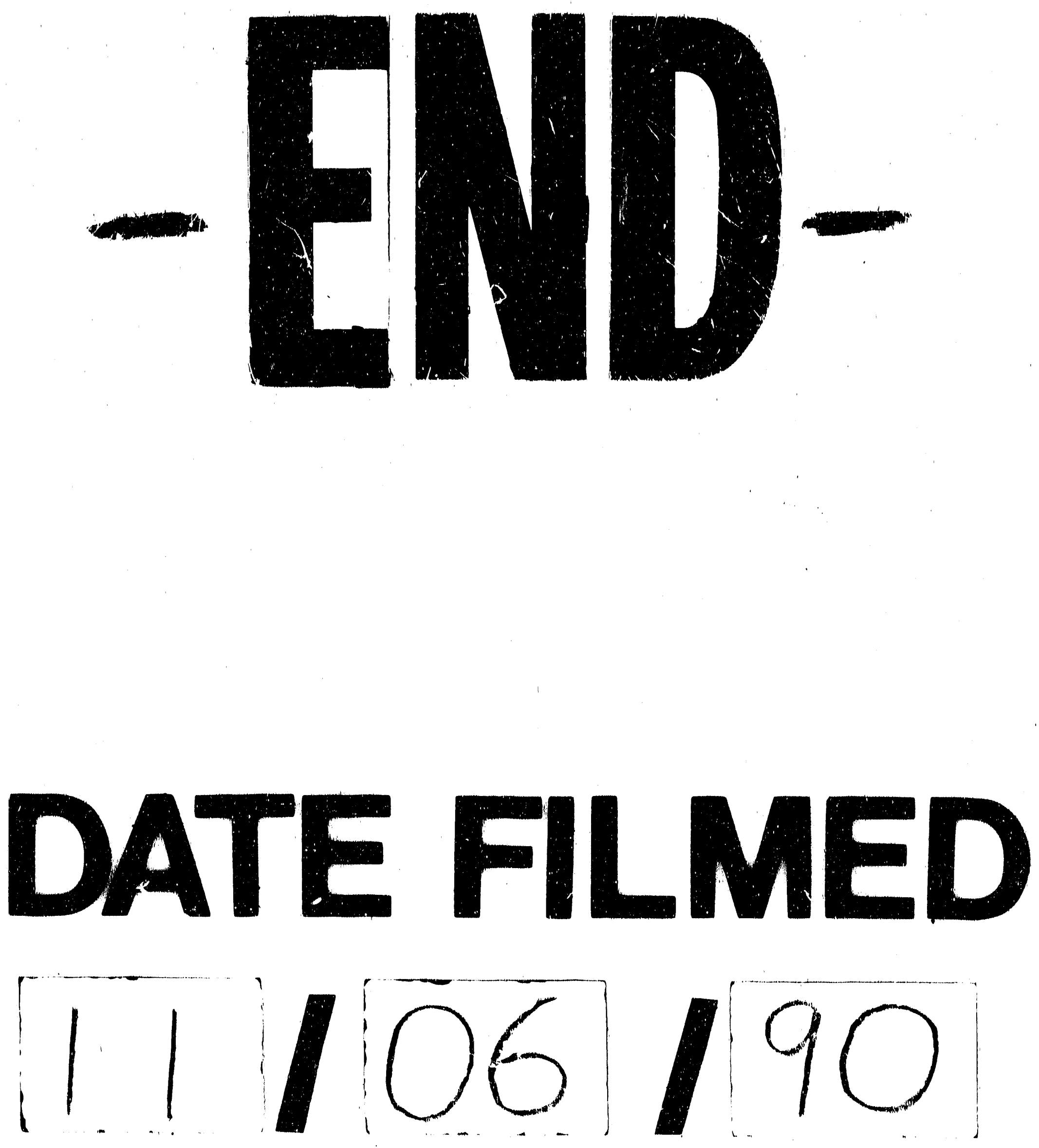
